OPEN ACCESS

Edited by:

Silvio Marchini

University of São Paulo, Brazil

Reviewed by:

Yash Veer Bhatnagar

Nature Conservation Foundation, India

Bruce Alexander Schulte,

Western Kentucky University,

United States

*Correspondence:

Rekha Warrie

reka.warrier@gmail.com

Specialty section:

This article was submitted to

Human-Wildlife Dynamics,

a section of the journa

Frontiers in Conservation Science

Received: 10 March 2021

Accepted: 08 July 2021

Published: 19 August 2021

Citation:

Warrier R, Noon BR and Bailey LL (2021) A Framework for Estimating Human-Wildlife Conflict Probabilities Conditional on Species Occupancy.

Front. Conserv. Sci. 2:679028.

doi: 10.3389/fcosc.2021.679028

\section{A Framework for Estimating Human-Wildlife Conflict Probabilities Conditional on Species Occupancy}

\author{
Rekha Warrier*, Barry R. Noon and Larissa L. Bailey \\ Department of Fish Wildlife and Conservation Biology, Colorado State University, Fort Collins, CO, United States
}

Managing human-wildlife conflicts (HWCs) is an important conservation objective for the many terrestrial landscapes dominated by humans. Forecasting where future conflicts are likely to occur and assessing risks to lives and livelihoods posed by wildlife are central to informing HWC management strategies. Existing assessments of the spatial occurrence patterns of HWC are based on either understanding spatial patterns of past conflicts or patterns of species distribution. In the former case, the absence of conflicts at a site cannot be attributed to the absence of the species. In the latter case, the presence of a species may not be an accurate measure of the probability of conflict occurrence. We present a Bayesian hierarchical modeling framework that integrates conflict reporting data and species distribution data, thus allowing the estimation of the probability that conflicts with a species are reported from a site, conditional on the species being present. In doing so, our model corrects for both false-positive and false-negative conflict reporting errors. We provide study design recommendations using simulations that explore the performance of the model under a range of conflict reporting probabilities. We applied the model to data on wild boar (Sus scrofa) space use and conflicts collected from the Central Terai Landscape (CTL), an important tiger conservation landscape in India. We found that tolerance for wildlife was a predictor of the probability with which farmers report conflict with wild boars from sites not used by the species. We also discuss useful extensions of the model when conflict data are verified for potential false-positive errors and when landscapes are monitored over multiple seasons.

Keywords: human-wildlife conflicts, species occupancy, integrated species distribution models, false-positive reporting errors, wild boar conflict

\section{INTRODUCTION}

World over, tropical, sub-tropical, temperate, and Arctic landscapes are experiencing increased anthropogenic pressures as a direct consequence of growth in human populations and demand for resources (Kennedy et al., 2019). The resulting increase in human activities in wildlife habitats is occurring alongside an increased recognition of the use of human-dominated areas by many adaptable wildlife species (Gordon, 2009; Ferreira et al., 2018). As a consequence of these shifts in habitat and resource use patterns by humans and wildlife populations, the frequency with which they interact is also increasing (Nyhus, 2016). Human-wildlife conflicts (HWC) are a subset of these interactions that adversely affect wildlife populations and human communities (Dickman, 2010). HWC can directly impact wildlife populations when they result in the removal of individuals 
either by legal or illegal means (Treves et al., 2006; Goswami et al., 2014). Persistent HWC, when unmitigated, can also affect conservation efforts by diminishing human tolerance for coexisting with wildlife (Kansky et al., 2016). Where human lives are lost, or where socio-economically marginalized communities are disproportionately affected, HWC also complicates the moral and ethical arguments in support of wildlife conservation. Preventing and mitigating HWC is therefore an imperative for the conservation of many threatened and endangered animal species. Consequently, monitoring HWC, and understanding its spatio-temporal dimensions and drivers is an integral part of most conservation programs.

Understanding the scale, nature, and drivers of HWC are fraught with challenges that arise both from the complexity of the process as well as biases inherent in observing the process. HWCs result from a complex interaction between social and ecological variables that may be heterogenous in time and space (Lischka et al., 2018). Foraging decisions made by animals are the underlying driver of the observed patterns of many forms of HWC (Hill, 2015). Foraging decisions typically involve tradeoffs between risks in accessing a food item and the net profitability of the food item (Baruch-Mordo et al., 2013; Blackwell et al., 2016). Long-term conflict prevention therefore depends upon clearly understanding what factors may be driving foraging decisions by a species. For example, a study of livestock depredation patterns by wolves in the Tibetan plateau found that while wolves occurred over a large portion of the landscape, attacks on livestock were more probable in areas of low ruggedness (Suryawanshi et al., 2013).

The probability of conflicts occurring with wildlife at any given location may be predictable given an understanding of a species' space-use patterns (Baruch-Mordo et al., 2014), or by investigating historic patterns of reported conflicts (Athreya et al., 2015). Space-use patterns of wildlife are commonly estimated by fitting species distribution models (SDMs) to occurrence or detection/non-detection data (Elith and Leathwick, 2009; Morelle and Lejeune, 2015; MacKenzie et al., 2017). Typically, SDMs estimate the probability with which bounded areas within a landscape are occupied or used by a species. However, space-use probabilities may not be an adequate reflection of the probability that conflicts with the species are likely to occur at a given site. For example, large carnivores such as tigers (Panthera tigris), lions (Panthera leo), and wolves (Canis lupus) have large home ranges that include human dominated areas, but conflicts with these species are often narrowly clustered in space and time (Treves et al., 2004; Gazzola et al., 2008; Packer et al., 2019; Warrier et al., 2020). Thus, HWC may occur within a small, non-random subset of the area over which wildlife and humans co-occur.

Patterns of HWC have been investigated extensively using distribution models applied to spatial location records of reported or observed conflicts (Karanth et al., 2013; Goswami et al., 2015; Miller, 2015). The state variable of interest in these models is the probability of a conflict event occurring at a given site. Historical records of conflict locations (Treves et al., 2004), newspaper reports of conflict events (Athreya et al., 2015); or self-reporting of conflicts by affected communities are typical sources of these data (Krafte Holland et al., 2018). These models provide insights into the spatial and temporal correlates of conflicts across a landscape and help delineate areas at high risk for conflict. In many of these efforts, the possibility of conflict events at a site going un-reported or un-detected, may lead to an underestimation of the probability of conflict occurrence (e.g., Goswami et al., 2015). Since these models do not provide estimates of the true distribution of the species in the landscape, it is not possible to determine if the absence of conflicts at a site is a consequence of the absence of the species at the site, or if the species occurred at the site without conflict. In the absence of this information, spatial models of conflict may not correctly identify the environmental factors most strongly associated with HWC, thereby limiting their applicability in forecasting where new conflicts are likely to occur (Treves et al., 2011). In addition, these models assume no false-positive reporting, that is, reporting of conflict from a site where the species does not occur or where conflicts are incorrectly attributed to a species. For example, a study evaluating reports of livestock depredation in Wisconsin found that out of the 575 cases where livestock owners blamed wolves for depredation, only $60 \%$ of the cases were actually attributable to wolves (Treves et al., 2002). False attribution of conflicts may be especially high for species for whom local community members have low tolerance (Suryawanshi et al., 2013; Dickman and Hazzah, 2016). Therefore, where the veracity of individual conflict reports is in question, the application of traditional distribution models may lead to positively biased estimates of the magnitude of conflicts.

We propose that a solution to these challenges lies in understanding the spatial patterns of conflicts as a function of a species' distribution, since species' presence at a site is a necessary condition for a potential HWC to occur at that site. Previous studies have attempted to link species distribution models with conflict risk models (e.g., Braunisch et al., 2011; de Souza et al., 2018). However, these studies did not explicitly link the probability of a species occurring at a site with conflict information, that is, explicitly estimating the probability of conflict occurring or being reported, conditional on species occupancy.

In the following, we describe a hierarchical Bayesian occupancy modeling approach to integrate data on species occurrence and conflict reports that may include false-positive errors. Our approach offers a generalized framework for estimating species occupancy and the probability of conflict being reported from a site conditional on species presence in situations where conflict data are not vetted for false-positive errors. After introducing the model, we provide study design recommendations based on a simulation study. We also illustrate the practical utility of the model by applying it to data on wild boar (Sus scrofa) distribution and human-wild boar conflict data generated by interviewing farmers. These data were collected from a human dominated agricultural corridor area within the Central Terai Landscape (CTL) in northern India, a globally significant tiger conservation unit (Wikramanayake et al., 2011).

This study was part of a larger effort to estimate habitat use of wild carnivores and herbivores within the agricultural corridor. Wild boar are an important tiger prey species (Hayward 
et al., 2012) and are widely regarded as an agricultural pest across their distributional range (Lewis et al., 2017). We explored how conflicts with wild boar are reported from sites occupied and unoccupied by the species. Specifically, we were interested in understanding how the attitudes of farmers toward wildlife influence their reporting of real and perceived conflicts with wild boar. We define "real" conflicts as any conflict report that is recorded from a site where the species is predicted to occur, whereas "perceived" conflicts are those that are reported from sites conditional on the species being absent. The spatial patterns of real conflicts are assumed to be associated with environmental factors that determine the distribution of wild boar and influence their foraging decisions. For example, in southern Italy, crop losses to wild boar typically occurred in areas dominated by shrubs and areas with intermediate forest cover (Francesco et al., 2014). On the contrary, perceived conflicts may be driven by the attitudes of community members toward the speciesfor example, community beliefs about the species potential to cause damage or the abundance of the species (Dickman, 2010; Dickman and Hazzah, 2016). Consequently, these two types of conflicts can be resolved only by the application of fundamentally different mitigation measures. The goal of our case study was to distinguish between real and perceived conflicts reports within the CTL, and understand how community attitudes toward wildlife and situational characteristics of the respondents, such as age and land holding size influence the reporting of these perceived conflicts. We discuss the implications of our findings as well as model extensions under scenarios where conflict data have been vetted for false-positive errors.

\section{MATERIALS AND METHODS}

Broadly, our models are similar to integrated species distribution models (Pacifici et al., 2017; Miller et al., 2019), where diverse data are integrated by means of a shared parameter. Specifically, our approach is a Bayesian hierarchical, false-positive occupancy model where data were generated under the single season, site confirmation design, employing multiple detection methods (Miller et al., 2011; Chambert et al., 2015; Clement, 2016). The site confirmation design using multiple detection methods involves the use of auxiliary detection methods that are free of false-positive errors to estimate the true occupancy probability of a site. For example, the multiple detection methods could include using interview surveys (ambiguous method) in conjunction with unambiguous methods such as sign surveys and camera surveys at the same survey locations (Warrier et al., 2020). Our model relies on two data sources. Data on conflicts with the species of interest are collected for $i=1,2, \ldots S$ sites or spatial units. At each site, conflict data are collected over $j=$ $1,2, \ldots J$ survey occasions. These data may be collected either through interviews of community members, or through conflict monitoring programs. If interviews of community members are used to generate data on conflicts for a given site, each interview respondent is treated as a survey occasion. Where data from conflict monitoring programs are used, survey occasions may represent discrete time intervals over which the presence or absence of a conflict event is recorded (Goswami et al., 2015). Additional data pertaining to each of these survey occasions may also be collected, such as information on prevailing weather and environmental covariates hypothesized to influence conflicts. For example, in the Greater Tsavo ecosystem in Kenya, the number of HWCs reported annually, and seasonally varied as a function of rainfall (Mukeka et al., 2020). These conflict surveys generate information on the total number of occasions within a site where a conflict event with the species of interest was reported. To predict the true underlying occupancy state for the target species at a given site, additional surveys for the species of interest are simultaneously conducted at all, or a subset, of the sites $(i=1$, $2, . . s ; s \leq S$ ) using a method assumed to be free of false-positive errors. Detection methods could include the use of camera traps, acoustic recorders, or sign surveys with surveys repeated $k=$ $1,2, . . K$ times at each site. Using the terminology associated with the site-confirmation, multiple detection methods design, we categorized the conflict surveys as a source of ambiguous data (i.e., they provide ambiguous information on whether the species occurs at a site) whereas the auxiliary surveys were assumed to result in unambiguous true detections at occupied sites.

\section{Model}

We adopted a Bayesian hierarchical modeling approach to predict patterns of species occupancy and the conditional probability of $\mathrm{HWC}$ reporting. The hierarchical model is composed of two sub-components, a process model, and an observation model. The process model describes the ecological processes that gave rise to the spatial patterns in the latent state variable (site occupancy). In the model, $z_{i}$ describes the occupancy state of a site " $i$ " for $S$ sites surveyed over a season. Within the survey season, sites are assumed to be closed to changes in their occupancy state. A site can be in one of two possible states, occupied $\left(z_{i}=1\right)$ or unoccupied $\left(z_{i}=0\right)$ by the target species. The occupancy state of a site " $i$ " $\left(z_{i}\right)$ is treated as a Bernoulli random variable with occupancy probability equal to $\psi_{\mathrm{i}}$. The parameter $\psi_{\mathrm{i}}$ describes the probability a site is occupied by the species of interest and may itself be defined as a function of site-specific covariates $x_{i}^{\prime}$. Here, $x_{i}^{\prime}$ was a vector of covariates characterizing the environment within site $i$.

$$
\begin{array}{r}
z_{i} \sim \operatorname{Bernoulli}\left(\Psi_{i}\right) \\
\operatorname{logit}\left(\Psi_{i}\right)=x_{i}^{\prime} \beta
\end{array}
$$

At site $i$, the total number of survey occasions where a conflict event is reported is represented by $w_{i}$. Similarly, $y_{i}$, represents the total number of detections of the species at a site $i$ using the unambiguous survey method. For occupied sites, the number of occasions during which conflicts with a species was recorded $\left(w_{i} \geq 0 \mid z_{i}=1\right)$ is described by a Binomial distribution with probability $p_{11}$. Whereas, at unoccupied sites, the number of occasions where conflict with the species of interest was reported $\left(w_{i} \geq 0 \mid z_{i}=0\right)$ follow a Binomial probability distribution with probability $p_{10}$. Thus, $p_{11}$ describes the probability a conflict with the species of interest was reported during a given survey at an occupied site. While $p_{10}$ represents the probability a conflict with 
a species was reported during a survey at an unoccupied site.

$$
w_{i} \sim\left\{\begin{array}{l}
\operatorname{Binom}\left(\mathrm{J}_{\mathrm{i}}, p_{10}\right) z_{i}=0 \\
\operatorname{Binom}\left(\mathrm{J}_{\mathrm{i}}, p_{11}\right) z_{i}=1
\end{array} \quad, \text { for } i \in \mathrm{S}\right.
$$

Similarly, using the unambiguous survey method (e.g., camera trap surveys), the total number of detections at an occupied site given " $K$ " survey occasions $\left(y_{i} \geq 0 \mid z_{i}=1\right)$ follow a Binomial probability distribution with detection probability equal to $r$.

$$
y_{i} \sim\left\{\begin{array}{cc}
0 & z_{i}=0 \\
\operatorname{Binom}\left(\mathrm{K}_{i}, r\right) & z_{i}=1
\end{array}, \text { for } i \in \mathrm{s} \subseteq \mathrm{S}\right.
$$

\section{Simulation Study}

We assessed the ability of our model to predict species occupancy $(\Psi)$ under a range of conditions. We focused on parameters associated with occupancy, since this was the state variable around which we integrated the space-use and conflict datasets. We simulated data for 100 sites exploring multiple scenarios involving alternative probabilities of falsely attributing conflicts to the focal species $\left(p_{10}\right)$ and true detection probabilities associated with the unambiguous survey method $(r)$. We considered three alternative possibilities for the probability that conflicts would be reported at least once at an unoccupied site $\left[p_{10}^{*}=1-\left(1-p_{10}\right)^{J} ; 0.1\right.$ (low), 0.5 (medium), 0.8 (high)]. We assumed that these probabilities remained constant across sites. For each level of $p_{10}^{*}$, we considered three scenarios for the probability of detecting the species at least once at a site using the unambiguous method $\left[r^{*}=1-(1-r)^{K} ; 0.1\right.$ (low), 0.5 (medium) and 0.8 (high)]. For each level of $p_{10}^{*}$; and $r^{*}$, we explored five alternate scenarios with respect to the proportion of sites where the unambiguous method was applied $(5,20,50$, 75 , and $100 \%)$. The result was 45 combinations of false-positive reporting and survey effort for the unambiguous method. The probability of a conflict reported at least once during a survey season from a site where the animal was predicted to occur $\left[p_{11}^{*}=\right.$ $\left.1-\left(1-p_{11}\right)^{J}\right]$ was set at 0.60 .

For all scenarios, we simulated site-specific occupancy probabilities as a function of a single covariate, logit $\left(\Psi_{i}\right)=\beta_{0}+$ $\beta_{1} * \operatorname{cov} v_{i}$. We assumed a mean occupancy probability of $25 \%\left(\beta_{0}\right.$ $=0.25$ ) and occupancy probabilities that declined with increasing values of a normally distributed covariate $\left(\beta_{1}=-0.60\right)$. Taken together, the scenarios represented the range of conflict reporting probabilities and survey effort for a hypothetical species characterized by low occupancy probability and moderately to high potential to be involved in conflicts where it occurred. For each scenario we fit our model to 100 simulated datasets and evaluated the precision of the estimated intercept $\left(\beta_{0}\right)$ and slope $\left(\beta_{1}\right)$ parameters. For all simulations, we used vague priors on model parameters by specifying a normal distribution with $\mu=0$ and $\sigma^{2}=2.5$. We generated 25,000 MCMC samples with a burnin period of 5,000 iterations. For all parameters modeled without covariates, we generated MCMC samples by sampling from their full conditional distributions via Gibb's sampling (Appendix A). For parameters modeled as functions of covariates, we used the Metropolis MCMC algorithm (Geyer, 1997). All simulations were carried out in the $\mathrm{R}$ statistical computing environment ( $\mathrm{R}$ Core Team, 2020).

\section{Application}

Data were collected from a $1,200 \mathrm{~km}^{2}$ agricultural corridor separating two tiger reserves in the CTL (Dudhwa Tiger Reserve and Pilibhit Tiger Reserve). We surveyed 46 randomly selected sites $(1.6 \times 1.6 \mathrm{~km}$ grid cells $)$ for wild boar and simultaneously collected data on human-wild boar conflicts between December 2015 and February 2016. We selected grid cells based on the Generalized Random Tessellation Stratified algorithm (GRTS; Stevens and Olsen, 2004). We collected detection data on wild boar by placing a single motion-activated camera (Cuddeback Attack) within the cell for a period of 40 days. The camera-trap surveys were a source of unambiguous data, assumed free of falsepositive errors. Ambiguous detection data were based on farmer interviews from a randomly selected subset of sites (27) where camera-trapping was conducted. We interviewed 1-10 farmers resident within each site using a survey instrument designed to generate data on conflict occurrence, and various, social, economic, and attitudinal correlates. For camera trap surveys, we treated each 5-day period as an occasion. For farmer interview surveys, each farmer was treated as a unique survey occasion.

Using the survey instrument, we obtained data on whether the respondent (farmer) was currently experiencing crop losses due to wildlife and what species they believed were responsible. We also recorded information on the age and land holding sizes of the respondents. In addition, we recorded the attitudes of respondents toward two ongoing conflict mitigation options: compensation for losses and fencing of protected areas. We also assessed the attitudes of respondents toward the prevailing legal restriction on lethally removing crop damaging wildlife species. Responses to these questions were recorded on a binary (Yes/No) scale.

We tested a number of hypotheses. (1) We a priori expected wild boar occupancy probabilities to decline with increasing distance to park boundaries (Karanth et al., 2013) - therefore, we modeled occupancy probability $(\psi)$ as a function of distance of the site to the nearest protected area boundary (PA). (2) We evaluated how the probability of a farmer reporting conflicts with wild boar at an unoccupied site $\left(p_{10}\right)$ was related to their tolerance for wildlife as reflected in their attitudes toward mitigation measures, and their attitude toward the legal restriction on lethal removal of problem animals. We hypothesized that farmers who favored compensation (Compensation) as a conflict mitigation strategy, and those who favored the removal of the legal restriction on lethal action (Hunt), would falsely report conflicts with higher probability (Dickman et al., 2011; Johnson et al., 2018). (3) We hypothesized that younger, less experienced farmers (Age) would have a higher probability of falsely reporting conflicts. (4) We evaluated the probability of a farmer reporting conflicts from a site occupied by wild boar $\left(p_{11}\right)$ as a function of their land holding size (LandOwned). We expected conflict reporting probabilities to decline with increasing farm size since crop depredation would have a more pronounced effect on small land holders. 
We tested the effects of distance to protected areas (PA) on occupancy probability, while modeling the remaining parameters without covariates (Table 1; Morin et al., 2020). Using the supported occupancy structure $(\triangle \mathrm{DIC}<5)$, we proceeded to identify best supported covariate structure for false conflict reporting p10 (probability of reporting conflicts from sites unoccupied by wild boar; Table 1), followed by evaluating the influence of land holding size on true conflict reporting, p11 (probability of reporting conflicts from sites occupied by wild boar; Table 1). We also fit a global model where all parameters are modeled as a function of covariates, $\psi(\mathrm{PA}) \mathrm{p} 10$ (Hunt)p11(LandOwned)r(.) as well as a model without covariates $\Psi() p .10() p .11() r.($.$) .$

All analyses were done in the $\mathrm{R}$ statistical computing environment (R Core Team, 2020). We specified vague priors on the model parameters and ran three chains with 50,000 MCMC samples each and used a burn-in period of 5000 iterations. We tested for chain convergence by calculating the Gelman-Rubin statistic for each parameter. We fit a total of six models and compared them based on their Deviance Information Criterion (DIC; Hooten and Hobbs, 2015).

\section{RESULTS}

\section{Simulation Results}

Under all 45 simulation scenarios, our model estimated parameters of interest with high precision. As expected, increasing unambiguous survey effort resulted in greater precision in estimates of the posterior means of $\beta_{0}$ and $\beta_{1}$. This result was observed both when a greater proportion of sites had unambiguous surveys and with an increase in detection probability $\left(r^{*}\right)$. Declines in precision were most pronounced with high levels of false-positive reporting $\left(p_{10}^{*}=0.80\right)$, when the unambiguous method had low detection probability $\left(r^{*}=\right.$ 0.10 ), or when unambiguous surveys occurred on fewer than $50 \%$ of the sites (Figure 1). These patterns of declining precision were more pronounced for the slope parameter $\left(\beta_{1}\right.$; Figure 1). Scenarios where the detection probability of the unambiguous method was low $\left(r^{*}=0.10\right)$, resulted in negatively biased

TABLE 1 | Candidate models and associated DIC values.

\begin{tabular}{lc}
\hline Model & DIC \\
\hline$\psi() p .10($ Hunt)p11(.)r(.) & 518.9 \\
$\psi() p .10($ Hunt)p11(LandOwned)r(.) & 522.02 \\
$\psi() p .10() p .11() r.()$. & 525.56 \\
$\psi() p .10($ Compensation)p11(.)r(.) & 526.25 \\
$\psi() p .10($ Age $)$ p11(.)r(.) & 527.33 \\
$\psi(\mathrm{PA}) \mathrm{p} 10($ Hunt)p11(LandOwned)r(.) & 688.86 \\
$\psi(\mathrm{PA}) \mathrm{p} 10(.) \mathrm{p} 11(.) \mathrm{r}()$. & 691.23 \\
\hline$\Psi$ is the probability the site was occupied by wild boar; $p 11$ is the probability that conflict \\
with wild boar was reported from a site occupied by the species; $p 10$ is the probability \\
that conflict with wild boar was reported from a site unoccupied by the species; and, $r$ \\
is the probability that wild boar were detected using camera traps at a site occupied by \\
the species.
\end{tabular}

estimates of mean occupancy and positively biased estimates of the slope parameter, irrespective of the level of survey effort and false-positive reporting probabilities. Across all scenarios and simulations, the estimated credible intervals included the true parameter value.

\section{Application Results}

Using camera traps, wild boar were detected at least once at nine sites. Conflicts with wild boar were reported at least once at 26 of the 27 sites based on interview surveys with farmers. Of the seven hypotheses tested (Table 1), a model where the probability of falsely reporting conflicts with wild boars at an unoccupied site was a function of attitudes in opposition to the restriction on lethal removal of wildlife (Hunt) had the highest support (lowest DIC). The model with the next highest support reflected our hypothesis that true conflict reporting probabilities would decline with increasing land holding sizes. Similarly, the model with the least support was one where both true and false conflict reporting probabilities were modeled without covariates and wild boar use probabilities were modeled as a function of distance of the cell to protected areas (PA).

Based on the best-supported model, wild boar habitat use across the surveyed sites was not a function of the distance to a protected area boundary. As per the model, wild boar used sampled sites with a mean probability of 0.24 (Figure 2). The probability of detecting wild boar using camera traps, given the site was occupied, was 0.33 for each 5 -day survey occasion (Figure 2). On average, the probability that a farmer would report conflicts from a site used by wild boar was 0.87 . In contrast, the probability that a farmer would report conflicts from a site not used by the species was 0.63 (Figure 2). Farmers who favored the removal of the legal restriction on hunting (Hunt) falsely reported conflicts with wild boar with a higher probability that those who favored the continuation of the law (Figure 3).

\section{DISCUSSION}

Our modeling approach allows for the joint estimation of species occupancy and the probability of reporting humanwildlife conflicts. In our model formulation, HWC reporting probabilities were estimated conditional on the presence or absence of the species at the site. This allowed for the estimation of the probability that conflicts may have been falsely recorded at an unoccupied site. The method we used provides a flexible modeling approach and offers a cost-effective and efficient strategy to monitor species space use and conflicts over large spatial scales. The model framework we used requires the collection of additional auxiliary data at all, or a subset, of the sites over which conflict monitoring was conducted. Our simulation results suggest that using auxiliary survey methods that are free of false positive errors with a cumulative detection probability $\left(r^{*}\right)>0.5$, results in unbiased estimates of covariate relationships. Conflict monitoring programs involving species for which human communities have low tolerance, or whose depredation patterns closely resemble those of other conspecific species (e.g., wolves and coyotes ; Treves et al., 2002), are likely 

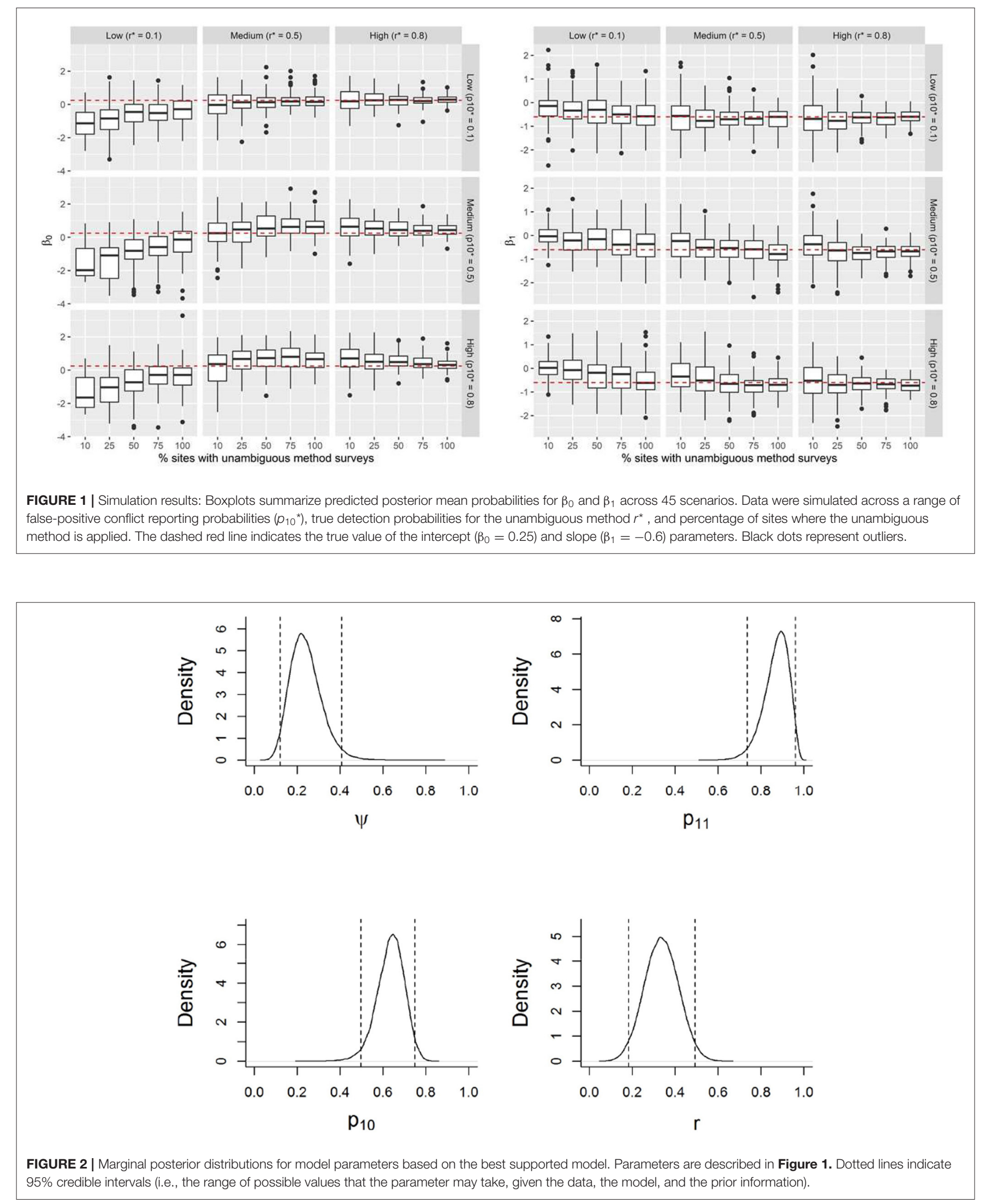


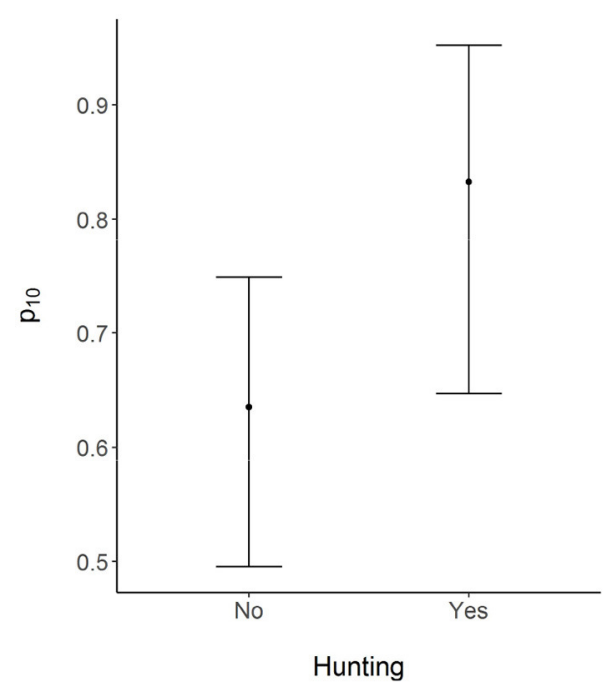

FIGURE 3 | Mean probability of falsely reporting conflicts with wild boar by respondents who did (Yes) and did not (No) favor removal of the prevailing ban on lethal removal of problem wildlife. Error bars represent $95 \%$ credible intervals.

circumstances where false reporting of conflicts may be high. In these situations, conducting auxiliary surveys at $\sim 50 \%$ of the sites over which conflict is being monitored are needed to discern the true rates and spatial distribution of conflicts (Figure 1). When false-positive reporting probabilities are very low, the proportion of sites where the auxiliary method should be applied depends on how true conflicts are reported at a site where the species occurs $\left(p_{11}\right)$ and the probability that the auxiliary survey method can detect the species when it occurs at a site $(r)$.

In our case study, farmers generally overestimated the magnitude of conflicts with wild boar. As discussed by Dickman (2010), perceived levels of conflicts are often shaped by multiple human factors in addition to the true impacts of the species. These factors include social (e.g., age, religion), psychological (e.g., attitudes, beliefs), situation (e.g., economic status), and other unresolved human-human conflicts that may manifest as reduced tolerance for wildlife.

Documenting when significant disparities exist between perceived and real conflicts is a first step toward increasing the tolerance or acceptance of the species in shared landscapes (Skupien et al., 2016). We found that community members who had a favorable attitude toward the lethal removal of wild animals by legal hunting were more likely to falsely report conflicts with wild boar (Figure 3). While we interpret attitudes toward the removal of the hunting restriction as reduced tolerance for wildlife, we emphasize that this relationship may be true only in the restricted context of our study. Hunting is a widespread and understudied issue affecting wildlife in India, and its character and underlying motivations vary widely across different regions of the country (Velho et al., 2012). Wild boar occurred in the surveyed area with a mean probability of 0.24 , yet community members widely perceived it as a significant agricultural pest, exemplified by the fact that conflicts with the species were reported from all the surveyed sites. In the absence of information on the actual distribution of wild boar, the conflict survey data would lead us to believe that the species is more widespread across the landscape. Similarly, in sites used by the species, there was a high probability of conflicts being reported by farmers. Results from our study, and a broader application of our survey modelling methods, has important consequences for the formulation of conflict mitigation measures (Anand and Radhakrishna, 2017). In the CTL, conflict mitigation should employ a two-pronged approach that includes compensation for crop losses based on defensible estimates of the actual probability of HWC, in conjunction with efforts to understand in greater detail community attitudes toward hunting and land sharing with wildlife.

An important caveat associated with our results is that our model cannot distinguish between true and false conflict reports from sites used by wild boars. Consequently, it is likely that within used sites, the intensity of conflict may be lower than our data and modeling suggests. We attempted to characterize contemporary conflicts with wild boar in the study area. Yet, the high rates of false-positive conflict reporting may be the result of respondents reporting long-standing conflicts rather than conflicts that occurred during the survey season. However, previous studies that relied on interview data to estimate wild boar distribution patterns have demonstrated the high prevalence of false-positive detection errors for the species (Pillay et al., 2014).

The modeling approach we adopted did not estimate the conditional probability of conflicts occurring at a site - rather, it estimated the conditional probability of conflicts being reported. Conflict reporting is conditional on occurrence only in situations where there are no false-positive reporting errors. However, if conflict reports can be verified and validated, the probability of conflict occurring, conditioned on a species' occupancy, can be estimated using a simple extension of our model. Our model can be extended by introducing an additional parameter $\delta_{i}$ representing the probability that a conflict event occurred at site $i$ occupied by the species of interest $\left(z_{i}=1\right)$. The latent state of the site with regards to conflict occurrence is given by $c_{i}$, such that $c_{i}=1$ when a conflict has occurred, and $c_{i}=0$ when no conflict occurred. The model then has the following form:

$$
\begin{aligned}
& z_{i} \sim \operatorname{Bernoulli}\left(\Psi_{i}\right) \\
& \operatorname{logit}\left(\Psi_{i}\right)=x_{i}^{\prime} \beta \\
& c_{i} \sim \operatorname{Bernoulli}\left(\delta_{i}^{*} z_{i}\right) \\
& y_{i} \sim\left\{\begin{array}{cc}
0 & z_{i}=0 \\
\operatorname{Binom}\left(\mathrm{K}_{i}, r_{i}\right) & z_{i}=1
\end{array}, \text { for } i \in s \subseteq S\right. \\
& w_{i} \sim\left\{\begin{array}{c}
0 \\
c_{i}=0 \\
\operatorname{Binom}\left(\mathrm{J}_{i}, p_{11}, r\right) \\
c_{i}=1
\end{array}, \text { for } i \in S\right.
\end{aligned}
$$

In this model, the probability of conflict occurring at a site where the species is present is given by $\Psi_{i} * \delta_{i}$. Like the occupancy parameter $\left(\Psi_{i}\right)$, conflict probability can be modeled as a function 
of covariates such that logit $\left(\delta_{i}\right)=u_{i}^{\prime} \alpha$, where $u_{i}$ is a vector of covariates associated with $i$ th site. The parameter $p_{11}$ represents the probability of detecting a conflict at a site where it has occurred. This is useful in situations where there is imperfect reporting of conflicts (Goswami et al., 2015). Where conflict detection is certain, the parameter $p_{11}$ can be interpreted as the intensity with which conflicts occurred at a site. When data are collected over multiple survey seasons, the model can be extended to represent the temporal dynamics of species space-use and the prevalence of conflict reports (Appendix B).

Understanding the link between a species' current distribution and conflict probability is essential for to effectively mitigate human-wildlife conflicts. For example, the willingness of people to coexist with a species is often linked to their perceptions of the risk that the species poses to their lives or livelihoods. Risk perception is reflected in the degree of tolerance which, in turn, determines the course of management actions directed toward a species (Riley and Decker, 2000; Knopff et al., 2016). In many cases, perceptions of risks are often disproportionately higher than the actual risks posed by the species. For example, a study of community perceptions of risks from cougars (Puma concolor) found that a large proportion of respondents incorrectly believed that the risks were higher than those incurred when using airplanes, automobiles, and tractors (Riley and Decker, 2000). Estimating the probability of HWC conditional on a species' current occupancy patterns is a way to estimate the true risks arising from land sharing with conflict-prone species. Our modeling approach provides a framework to estimate conflicts and conflict reporting as a function of species occupancy while simultaneously accounting for false-positive reporting errors. Importantly, the survey, and analysis approach we propose can be implemented with minimal additional survey effort and provide defensible estimates of the magnitude of HWCs.

\section{DATA AVAILABILITY STATEMENT}

The raw data supporting the conclusions of this article will be made available by the authors, without undue reservation.

\section{REFERENCES}

Anand, S., and Radhakrishna, S. (2017). Investigating trends in human-wildlife conflict: is conflict escalation real or imagined? J. Asia-Pacific Biodivers. 10, 154-161. doi: 10.1016/j.japb.2017.02.003

Athreya, V., Srivathsa, A., Puri, M., Karanth, K. K., Kumar, N. S., and Karanth, K. U. (2015). Spotted in the news: using media reports to examine leopard distribution, depredation, and management practices outside protected areas in Southern India. PLoS ONE 10:e0142647. doi: 10.1371/journal.pone.0 142647

Baruch-Mordo, S., Webb, C. T., Breck, S. W., and Wilson, K. R. (2013). Use of patch selection models as a decision support tool to evaluate mitigation strategies of human-wildlife conflict. Biol. Conserv. 160, 263-271. doi: 10.1016/j.biocon.2013. 02.002

Baruch-Mordo, S., Wilson, K. R., Lewis, D. L., Broderick, J., Mao, J. S., and Breck, S. W. (2014). Stochasticity in natural forage production

\section{ETHICS STATEMENT}

The studies involving human participants were reviewed and approved by Colorado State University IRB. Written informed consent for participation was not required for this study in accordance with the national legislation and the institutional requirements.

\section{AUTHOR CONTRIBUTIONS}

RW lead the study design, data collection, model, and manuscript development. $\mathrm{BN}$ and $\mathrm{LB}$ made substantial contributions to study design, model, and manuscript development. All authors approved the submitted version.

\section{FUNDING}

The study was funded by Grants from WWF-India, the Center for Collaborative Conservation (Colorado State University) and The Rufford Foundation.

\section{ACKNOWLEDGMENTS}

Permission for the study was granted by the Field Director of Dudhwa Tiger Reserve, Uttar Pradesh State forest Department. The model was co-developed with Lanier. W. We are grateful for feedback on the model from M. Hooten. P. Chanchani supported the study by providing camera-traps. We thank Landau.V for providing important insights during model development. Raja, Rambharose, and M. Singh for help with data collection. We are grateful for collaboration and support from S. Worah, D. Ghose, J. Vattakaven, H. Karandikar, Y. Shethia, M. Gupta, and D. Hassan at WWF-India, K. Crooks, and T. Teel at Colorado State University.

\section{SUPPLEMENTARY MATERIAL}

The Supplementary Material for this article can be found online at: https://www.frontiersin.org/articles/10.3389/fcosc. 2021.679028/full\#supplementary-material

affects use of urban areas by black bears: implications to management of human-bear conflicts. PLOS ONE 9:e85122. doi: 10.1371/journal.pone.00 85122

Blackwell, B. F., DeVault, T. L., Fernández-Juricic, E., Gese, E. M., Gilbert-Norton, L., and Breck, S. W. (2016). No single solution: application of behavioural principles in mitigating human-wildlife conflict. Anim. Behav. 120, 245-254. doi: 10.1016/j.anbehav.2016.07.013

Braunisch, V., Patthey, P., and Arlettaz, R. (2011). Spatially explicit modeling of conflict zones between wildlife and snow sports: prioritizing areas for winter refuges. Ecol. Appl. 21, 955-967. doi: 10.1890/09-2167.1

Chambert, T., Miller, D. A. W., and Nichols, J. D. (2015). Modeling false positive detections in species occurrence data under different study designs. Ecology 96, 332-339. doi: 10.1890/14-1507.1

Clement, M. J. (2016). Designing occupancy studies when false-positive detections occur. Methods Ecol. Evol 7, 1538-1547 doi: 10.1111/2041-210X.12617

de Souza, J. C., da Silva, R. M., Gonçalves, M. P. R., Jardim, R. J. D., and Markwith, S. H. (2018). Habitat use, ranching, and human-wildlife conflict within a 
fragmented landscape in the Pantanal, Brazil. Biol. Conserv. 217, 349-357. doi: 10.1016/j.biocon.2017.11.019

Dickman, A. J. (2010). Complexities of conflict: the importance of considering social factors for effectively resolving human-wildlife conflict. Anim. Conserv. 13, 458-466. doi: 10.1111/j.1469-1795.2010.00368.x

Dickman, A. J., and Hazzah, L. (2016). "Money, myths and man-eaters: Complexities of human-wildlife conflict," in Problematic Wildlife: A Cross-Disciplinary Approach (New York, NY: Springer International Publishing), 339-356.

Dickman, A. J., Macdonald, E. A., and Macdonald, D. W. (2011). A review of financial instruments to pay for predator conservation and encourage human-carnivore coexistence. Proc. Natl. Acad. Sci. U.S.A. 108, 13937-13944. doi: 10.1073/pnas.1012972108

Elith, J., and Leathwick, J. R. (2009). Species distribution models: ecological explanation and prediction across space and time. Annu. Rev. Ecol. Evol. Syst. 40, 677-697. doi: 10.1146/annurev.ecolsys.110308.120159

Ferreira, A. S., Peres, C. A., Bogoni, J. A., and Cassano, C. R. (2018). Use of agroecosystem matrix habitats by mammalian carnivores (Carnivora): a global-scale analysis. Mamm. Rev. 48, 312-327. doi: 10.1111/mam.12137

Francesco, F. G., Bonardi, A., Mairota, P., Leronni, V., and Padoa-schioppa, E. (2014). Predicting wild boar damages to croplands in a mosaic of agricultural and natural areas. Curr. Zool. 60, 172-179. doi: 10.1093/czoolo/60.2.170

Gazzola, A., Capitani, C., Mattioli, L., and Apollonio, M. (2008). Livestock damage and wolf presence. J. Zool. 274, 261-269. doi: 10.1111/j.1469-7998.2007.00381.x

Geyer, C. . (1997). "Introduction to markov Chain Monte Carlo," in Handbook of Markov Chain Monte Carlo, eds S. Brooks, A. Gelman, G. Jones, and X. L. Meng (Boca Raton, FL: Chapman and Hall/CRC), 3-48.

Gordon, I. J. (2009). What is the future for wild, large herbivores in humanmodified agricultural landscapes? Wildlife Biol. 15, 1-9. doi: 10.2981/06-087

Goswami, V. R., Medhi, K., Nichols, J. D., and Oli, M. K. (2015). Mechanistic understanding of human-wildlife conflict through a novel application of dynamic occupancy models. Conserv. Biol. 29, 1100-1110. doi: $10.1111 /$ cobi.12475

Goswami, V. R., Vasaudev, D., and Oli, M. K. (2014). The importance of conflcitinduced mortality for conservation planning in areas of human-elephant cooccurence. Biol. Conserv. 176, 191-198. doi: 10.1016/j.biocon.2014.05.026

Hayward, M. W., Jedrzejewski, W., and Jêdrzejewska, B. (2012). Prey preferences of the tiger Panthera tigris. J. Zool. 286, 221-231. doi: 10.1111/j.1469-7998.2011.00871.x

Hill, C. M. (2015). Perspectives of "Conflict" at the wildlife-agriculture boundary: 10 Years On. Hum. Dimens. Wildl. 20, 296-301. doi: $10.1080 / 10871209.2015 .1004143$

Hooten, M. B., and Hobbs, N. T. (2015). A guide to Bayesian model selection for ecologists. Ecol. Monogr. 85, 3-28. doi: 10.1890/14-0661.1

Johnson, M., Karanth, K., and Weinthal, E. (2018). Compensation as a policy for mitigating human-wildlife conflict around four protected areas in Rajasthan, India. Conserv. Soc. 16:305. doi: 10.4103/cs.cs_17_1

Kansky, R., Kidd, M., and Knight, A. T. (2016). A wildlife tolerance model and case study for understanding human wildlife conflicts. Biol. Conserv. 201, 137-145. doi: 10.1016/j.biocon.2016.07.002

Karanth, K. K., Gopalaswamy, A. M., Prasad, P. K., and Dasgupta, S. (2013). Patterns of human-wildlife conflicts and compensation: insights from Western Ghats protected areas. Biol. Conserv. 166, 175-185. doi: 10.1016/j.biocon.2013.06.027

Kennedy, C. M., Oakleaf, J. R., Theobald, D. M., Baruch-Mordo, S., and Kiesecker, J. (2019). Managing the middle: a shift in conservation priorities based on the global human modification gradient. Glob. Chang. Biol. 25, 811-826. doi: $10.1111 /$ gcb. 14549

Knopff, A. A., Knopff, K. H. and St. Clair, C. C. (2016). Tolerance for cougars diminished by high perception of risk. Ecol. Soc. 21:33. doi: 10.5751/ES-08933-210433

Krafte Holland, K., Larson, L. R., and Powell, R. B. (2018). Characterizing conflict between humans and big cats Panthera spp: a systematic review of research trends and management opportunities. PLoS ONE 13:e0203877. doi: 10.1371/journal.pone. 0203877

Lewis, J. S., Farnsworth, M. L., Burdett, C. L., Theobald, D. M., Gray, M., and Miller, R. S. (2017). Biotic and abiotic factors predicting the global distribution and population density of an invasive large mammal. Sci. Rep. 7:44152. doi: $10.1038 /$ srep44152

Lischka, S. A., Teel, T. L., Johnson, H. E., Reed, S. E., Breck, S., Don Carlos, A., et al. (2018). A conceptual model for the integration of social and ecological information to understand human-wildlife interactions. Biol. Conserv. 225, 80-87. doi: 10.1016/j.biocon.2018.06.020

MacKenzie, D. I., Nichols, J. D., Royle, J. A., Pollock, K. H., Bailey, L., and Hines, J. E. (2017). Occupancy Estimation and Modeling: Inferring Patterns and Dynamics of Species Occurrence. Amsterdam: Elsevier.

Miller, D. A., Nichols, J. D., McClintock, B. T., Grant, E. H. C., Bailey, L. L., and Weir, L. A. (2011). Improving occupancy estimation when two types of observational error occur: non-detection and species misidentification. Ecology 92, 1422-1428. doi: 10.1890/10-1396.1

Miller, D. A. W., Pacifici, K., Sanderlin, J. S., and Reich, B. J. (2019). The recent past and promising future for data integration methods to estimate species' distributions. Methods Ecol. Evol. 10, 22-37. doi: 10.1111/2041-210X.13110

Miller, J. R. B. (2015). Mapping attack hotspots to mitigate human-carnivore conflict: approaches and applications of spatial predation risk modeling. Biodivers. Conserv. 24, 2887-2911. doi: 10.1007/s10531-015-0993-6

Morelle, K., and Lejeune, P. (2015). Seasonal variations of wild boar Sus scrofa distribution in agricultural landscapes: a species distribution modelling approach. Eur. J. Wildl. Res. 61, 45-56. doi: 10.1007/s10344-014-0872-6

Morin, D. J., Yackulic, C. B., Diffendorfer, J. E., Lesmeister, D. B., Nielsen, C. K., Reid, J., et al. (2020). Is your ad hoc model selection strategy affecting your multimodel inference? Ecosphere 11:e02997. doi: 10.1002/ecs2.2997

Mukeka, J. M., Ogutu, J. O., Kanga, E. and Røskaft, E (2020). Spatial and temporal dynamics of human-wildlife conflicts in the Kenya Greater Tsavo Ecosystem. Hum. Wildife Interact. 14, 255-272. doi: 10.26077/bf21-497e

Nyhus, P. J. (2016). Human-wildlife conflict and coexistence. Annu. Rev. Environ. Resour. 41, 143-171. doi: 10.1146/annurev-environ-110615-085634

Pacifici, K., Reich, B. J., Miller, D. A. W., Gardner, B., Stauffer, G., Singh, S., et al. (2017). Integrating multiple data sources in species distribution modeling: a framework for data fusion*. Ecology 98, 840-850. doi: 10.1002/ecy.1710

Packer, C., Shivakumar, S., Athreya, V., Craft, M. E., Dhanwatey, H., Dhanwatey, P., et al. (2019). Species-specific spatiotemporal patterns of leopard, lion and tiger attacks on humans. J. Appl. Ecol. 56, 585-593. doi: 10.1111/1365-2664.13311

Pillay, R., Miller, D. A. W., Hines, J. E., Joshi, A. A., and Madhusudan, M. D. (2014). Accounting for false positives improves estimates of occupancy from key informant interviews. Divers. Distrib. 20, 223-235. doi: 10.1111/ddi.0.12151

R Core Team (2020). A Language and Environment for Statistical Computing. Available online at: https://www.r-project.org/

Riley, S., and Decker, D. (2000). Wildlife stakeholder acceptance capacity for cougars in Montana. Wildl. Soc. Bull. 28, 931-939. doi: $10.1080 / 10871200009359187$

Skupien, G. M., Andrews, K. M., and Larson, L. R. (2016). Teaching tolerance? Effects of conservation education programs on wildlife acceptance capacity for the American alligator. Hum. Dimens. Wildl. 21, 264-279. doi: 10.1080/10871209.2016.1147624

Stevens, D. L., and Olsen, A. R. (2004). Spatially balanced sampling of natural resources. J. Am. Stat. Assoc. 99, 262-278. doi: 10.1198/016214504000000250

Suryawanshi, K. R., Bhatnagar, Y. V., Redpath, S., and Mishra, C. (2013). People, predators and perceptions: patterns of livestock depredation by snow leopards and wolves. J. Appl. Ecol. 50, 550-560. doi: 10.1111/1365-2664.12061

Treves, A., Jurewicz, R. R., Naughton-Treves, L., Rose, R. A., Willging, R. C., and Wydeven, A. P. (2002). Wolf depredation on domestic animals in Wisconsin, 1976-2000. Wildl. Soc. Bull. 30, 231-241.

Treves, A., Martin, K. A., Wydeven, A. P., and Wiedenhoeft, J. E. (2011). Forecasting environmental hazards and the application of risk maps to predator attacks on livestock. Bioscience 61, 451-458. doi: 10.1525/bio.2011.61.6.7

Treves, A., Naughton-Treves, L., Harper, E. K., Mladenoff, D. J., Rose, R. A., Sickley, T. A., et al. (2004). Predicting human-carnivore conflict: a spatial model derived from 25 years of data on wolf predation on livestock. Conserv. Biol. 18, 114-125. doi: 10.1111/j.1523-1739.2004.00189.x

Treves, A., Wallace, R. B., Naughton-Treves, L., and Morales, A. (2006). Comanaging human-wildlife conflicts: a review. Hum. Dimens. Wildl. 11, 383-396. doi: 10.1080/10871200600984265 
Velho, N., Karanth, K. K., and Laurence, W. F. (2012). Hunting: A serious and understudied threat in India, a globally significant conservation region. Biol Conserv 148, 210-215 doi: 10.1016/j.biocon.2012.01.022

Warrier, R., Noon, B. R., and Bailey, L. (2020). Agricultural lands offer seasonal habitats to tigers in a human-dominated and fragmented landscape in India. Ecosphere 11: e03080. doi: 10.1002/ecs2.3080

Wikramanayake, E., Dinerstein, E., Seidensticker, J., Lumpkin, S., Pandav, B., Shrestha, M., et al. (2011). A landscape-based conservation strategy to double the wild tiger population. Conserv. Lett. 4, 219-227. doi: 10.1111/j.1755-263X.2010.00162.x

Conflict of Interest: The authors declare that the research was conducted in the absence of any commercial or financial relationships that could be construed as a potential conflict of interest.
Publisher's Note: All claims expressed in this article are solely those of the authors and do not necessarily represent those of their affiliated organizations, or those of the publisher, the editors and the reviewers. Any product that may be evaluated in this article, or claim that may be made by its manufacturer, is not guaranteed or endorsed by the publisher.

Copyright (c) 2021 Warrier, Noon and Bailey. This is an open-access article distributed under the terms of the Creative Commons Attribution License (CC BY). The use, distribution or reproduction in other forums is permitted, provided the original author(s) and the copyright owner(s) are credited and that the original publication in this journal is cited, in accordance with accepted academic practice. No use, distribution or reproduction is permitted which does not comply with these terms. 